\title{
Dynamic research of a nonlinear stochastic vibratory machine
}

\author{
Yimin Zhang ${ }^{\mathrm{a}}$, Qiaoling Liu ${ }^{\mathrm{a}}$ and Bangchun Wen ${ }^{\mathrm{b}}$ \\ ${ }^{a}$ Department of Mechanics, Jilin University, Changchun, 130025, P.R. China \\ ${ }^{\mathrm{b}}$ School of Mechanical Engineering, Northeastern University, Shenyang, 110004, P.R. China
}

Received 18 June 2001

Revised 28 December 2001

\begin{abstract}
This paper presents the dynamics problems of stochastic vibratory machine systems. The random responses of the vibratory machine systems with stochastic parameters subjected to random excitation are researched using a stochastic perturbation method. The numerical results are obtained. The dynamic characteristics of nonlinear stochastic vibratory machine are analyzed.
\end{abstract}

\section{Introduction}

The vibratory machines are widely employed in the many industry fields, such as mine and metallurgy etc.. During the last three decades, there has been considerable research activity in the area of engineering research. A substantial of development of the vibratory machines has taken place rapidly and evidently [1-8]. Since the manufacturing error, measurement error of mechanical systems, the respective materials and geometric characteristics, all have randomness, it is very important to investigate uncertain vibratory machine problem. In the theory of random vibration, the mechanical systems are generally expressed as deterministic model and the external input is treated as a stochastic process to analyze the statistic response. But uncertainty of system parameters is inherent in most engineering problems, and its effects on mechanical response should be assessed.

It is clear that stochastic analysis requires information of the distributions, or joint distribution. In practice they are often unavailable or difficult to be determined from insufficient data. Furthermore, even when the required distributions can be specified, it is still difficult to exercise relevant numerical integration. But the method presented in this paper is independent of those distributions, so it is natural that there is no more any difficulty with such distributions as well as their integration. As long as the first order moment and second order moment of mechanical random parameters are known, the first order moment and second order moment of responses can be determined. This paper presents an approximate solution for stochastic vibratory machines. The system is a multi-degree-offreedom nonlinear random vibratory system with random parameters. Stochastic perturbation technique is employed to systematically the random responses of the vibratory machines. This formulation is easily amenable to computational procedures.

\section{Nonlinear random vibration mechanical system}

The nonlinear stiffness and damping are generally used in the vibratory machine. There are many advantages in the nonlinear mechanism relative to the linear mechanism. The nonlinear two-mass vibratory machine shown in Fig. 1. The stochastic differential equation is represented as

$$
\left.\begin{array}{l}
m_{1} \ddot{x}_{1}+c \dot{x}_{1}-c \dot{x}_{2}+\left(k_{0}+k\right) x_{1} \\
\quad-\left(k_{0}+k\right) x_{2}+\varphi(\dot{\boldsymbol{x}}, \boldsymbol{x})=k_{0} r \sin \omega t \\
m_{2} \ddot{x}_{2}-c \dot{x}_{1}+c \dot{x}_{2}-\left(k_{0}+k\right) x_{1} \\
\quad+\left(k_{0}+k\right) x_{2}-\varphi(\dot{\boldsymbol{x}}, \boldsymbol{x})=-k_{0} r \sin \omega t
\end{array}\right\}
$$

where $m_{1}$ and $m_{2}$ are two-masses, $c$ is damping coefficient, $k_{0}$ is spring stiffness of connecting rod, $k$ is 


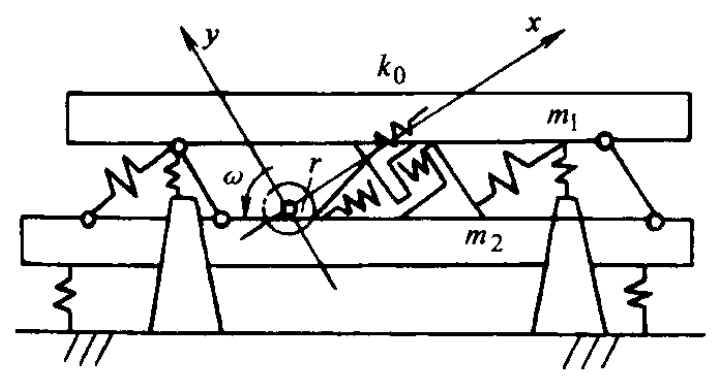

a)

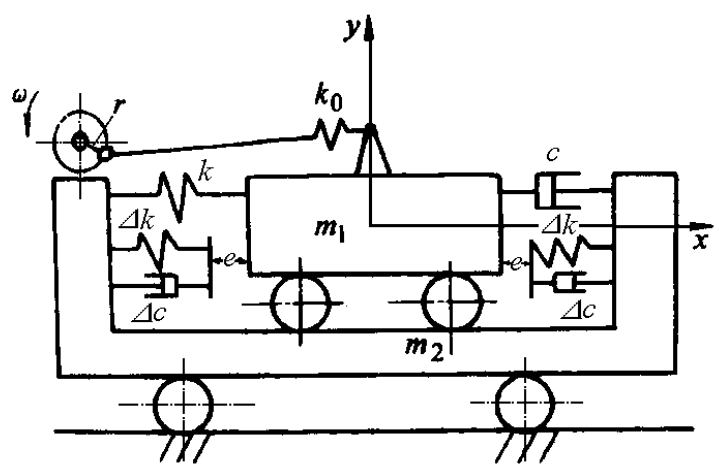

b)

Fig. 1. Nonlinear vibratory machine. (a) Actual work mechanism; (b) Mechanical model.

spring stiffness of main vibration system. $\omega$ is the frequency of main shaft, $r$ is the eccentricity of main shaft. $t$ is time, $x_{1}, x_{2}, \dot{x}_{1}, \dot{x}_{2}, \ddot{x}_{1}, \ddot{x}_{2}$ are the displacements, velocities, accelerations of mass 1 and mass 2. $\varphi(\dot{\boldsymbol{x}}, \boldsymbol{x})$ represents nonlinear damping and stiffness, namely

$$
\begin{gathered}
\varphi(\dot{\boldsymbol{x}}, \boldsymbol{x}) \\
=\left\{\begin{array}{c}
0 \quad-e \leqslant x_{1}-x_{2} \leqslant e \\
\Delta c\left(\dot{x}_{1}-\dot{x}_{2}\right)+\Delta k\left(x_{1}-x_{2}-e\right) \\
e \leqslant x_{1}-x_{2} \leqslant \infty \\
\Delta c\left(\dot{x}_{1}-\dot{x}_{2}\right)+\Delta k\left(x_{1}-x_{2}+e\right) \\
-\infty \leqslant x_{1}-x_{2} \leqslant-e
\end{array}\right.
\end{gathered}
$$

where $\Delta c$ and $\Delta k$ are damping and stiffness coefficients of interval spring. $e$ is the interval of the spring. In engineering, the masses are mostly assumed to be deterministic, and the probabilistic effects are described through the random parameters of spring stiffness and damping.

\section{Stochastic perturbation technique}

In the recent years much research has been done to quantify uncertainties in engineering systems and their combined effect on the response. The stochastic perturbation techniques have proven to be efficient in nonlinear random vibration mechanical system. A major advantage of these techniques is that the joint probability density or distribution functions need not be known, but only the first two moments.

Consider the motion of a nonlinear multi-degree-offreedom system with random parameters subjected to the random excitations and described by the differential equation

$$
\boldsymbol{M} \ddot{\boldsymbol{x}}+\boldsymbol{f}(\boldsymbol{R}, \boldsymbol{x}, \dot{\boldsymbol{x}})=\boldsymbol{F}(\boldsymbol{R}, t)
$$

where $\boldsymbol{M}, \boldsymbol{f}, \boldsymbol{x}$ and $\boldsymbol{F}$ are the generalized mass, internal force, displacement, and external force, respectively; and a superscript dot represents the time $(t)$ derivative. The probabilistic effects are described through the random parameter vector $\boldsymbol{R}=$ $\left(r_{1}, r_{2}, \ldots, r_{q}\right)^{T}$ of order $q$, which can include the probabilistic distributions of all random variable properties. Obviously, Eq. (3) is a vector-valued and matrixvalued function equation.

The random parameter $r_{i}$, mass matrix $M$, internal force vector $\boldsymbol{f}$, displacement vector $\boldsymbol{x}$, velocity vector $\dot{\boldsymbol{x}}$, acceleration vector $\ddot{\boldsymbol{x}}$, and external force vector $\boldsymbol{F}$ are expanded as

$$
\begin{aligned}
r_{i} & =r_{i d}+\varepsilon r_{i r}, \boldsymbol{M}=\boldsymbol{M}_{d}+\varepsilon \boldsymbol{M}_{r}, \\
\boldsymbol{f} & =\boldsymbol{f}_{d}+\varepsilon \boldsymbol{f}_{r}, \boldsymbol{x}=\boldsymbol{x}_{d}+\varepsilon \boldsymbol{x}_{r}, \\
\dot{\boldsymbol{x}} & =\dot{\boldsymbol{x}}_{d}+\varepsilon \dot{\boldsymbol{x}}_{r}, \ddot{\boldsymbol{x}}=\ddot{\boldsymbol{x}}_{d}+\varepsilon \ddot{\boldsymbol{x}}_{r} \\
\boldsymbol{F} & =\boldsymbol{F}_{d}+\varepsilon \boldsymbol{F}_{r}
\end{aligned}
$$

where $\varepsilon$ is a small parameter, here $\varepsilon$ is used to determine the order of formulated equations. The part that expressed by subscript $d$ is certain part in the random parameters and the part that expressed by subscript $r$ is the random part with zero mean value in the random parameters. Obviously, it is required that the values of the random part are much smaller than the values of the certain part. Both sides of Eq. (4) is evaluated about the mean value of random variables as follows

$$
\begin{aligned}
& E\left(r_{i}\right)=E\left(r_{i d}\right)+\varepsilon E\left(r_{i r}\right)=r_{i d} \\
& E(\boldsymbol{x})=E\left(\boldsymbol{x}_{d}\right)+\varepsilon E\left(\boldsymbol{x}_{r}\right)=\boldsymbol{x}_{d}, \\
& E(\dot{\boldsymbol{x}})=E\left(\dot{\boldsymbol{x}}_{d}\right)+\varepsilon E\left(\dot{\boldsymbol{x}}_{r}\right)=\dot{\boldsymbol{x}}_{d}, \\
& E(\ddot{\boldsymbol{x}})=E\left(\ddot{\boldsymbol{x}}_{d}\right)+\varepsilon E\left(\ddot{\boldsymbol{x}}_{r}\right)=\ddot{\boldsymbol{x}}_{d}
\end{aligned}
$$


Similarly, both sides of Eq. (4) are evaluated taking the variance of random variables as follows

$$
\begin{aligned}
& \operatorname{Cov}\left(r, r_{j}\right)=\varepsilon^{2} E\left(r_{i r} r_{j r}\right) \\
& \operatorname{Cov}(\boldsymbol{x})=\varepsilon^{2} E\left(\boldsymbol{x}, \boldsymbol{x}_{r}^{T}\right), \\
& \operatorname{Cov}(\dot{\boldsymbol{x}})=\varepsilon^{2} E\left(\dot{\boldsymbol{x}}_{r} \dot{\boldsymbol{x}}_{r}^{T}\right), \\
& \operatorname{Cov}(\ddot{\boldsymbol{x}})=\varepsilon^{2} E\left(\ddot{\boldsymbol{x}}_{r} \ddot{\boldsymbol{x}}_{r}^{T}\right)
\end{aligned}
$$

Substituting Eq. (4) into Eq. (3), yields

$$
\begin{aligned}
& \left(\boldsymbol{M}_{d}+\varepsilon \boldsymbol{M}_{r}\right)\left(\ddot{\boldsymbol{x}}_{d}+\varepsilon \ddot{\boldsymbol{x}}_{r}\right)+\boldsymbol{f}_{d}(\boldsymbol{R}, \boldsymbol{x}, \dot{\boldsymbol{x}}) \\
+ & \varepsilon \boldsymbol{f}_{r}(\boldsymbol{R}, \boldsymbol{x}, \dot{\boldsymbol{x}})=\boldsymbol{F}_{d}(\boldsymbol{R}, r)+\varepsilon \boldsymbol{F}_{r}(\boldsymbol{R}, t)
\end{aligned}
$$

Expanding the above equation, and equaling the terms of like powers of $\varepsilon$, we obtain

$$
\begin{array}{rrr}
\varepsilon^{0} & \boldsymbol{M}_{d} \ddot{\boldsymbol{x}}_{d}+\boldsymbol{f}_{d}(\boldsymbol{R}, \boldsymbol{x}, \dot{\boldsymbol{x}})= & \boldsymbol{F}_{d}(\boldsymbol{R}, t) \\
\varepsilon^{1} \quad \boldsymbol{M}_{d} \ddot{\boldsymbol{x}}_{r}+\boldsymbol{f}_{r}(\boldsymbol{R}, \boldsymbol{x}, \dot{\boldsymbol{x}})= & \boldsymbol{F}_{r}(\boldsymbol{R}, t) \\
& -\boldsymbol{M}_{r} \ddot{\boldsymbol{x}}_{d}
\end{array}
$$

The solutions for the certain part, namely $\boldsymbol{x}_{d}, \dot{\boldsymbol{x}}_{d}, \ddot{\boldsymbol{x}}_{d}$, the mean values of the responses are studied from Eq. (10). The mean values of the random responses are represented as

$$
E(\boldsymbol{x})=\boldsymbol{x}_{d}, E(\dot{\boldsymbol{x}})=\dot{\boldsymbol{x}}_{d}, E(\ddot{\boldsymbol{x}})=\ddot{\boldsymbol{x}}_{d},
$$

For determining the variances and covariances, $\boldsymbol{M}_{r}$, $\boldsymbol{f}_{r}, \boldsymbol{x}_{r}, \dot{\boldsymbol{x}}_{r}, \ddot{\boldsymbol{x}}_{d}$ and $\boldsymbol{F}_{r}$ are expanded about the mean values $\overline{\boldsymbol{R}}$ of the random parameters in the first approximation via Taylor series, namely,

$$
\begin{gathered}
\boldsymbol{M}_{r}=\sum_{i=1}^{q} \frac{\partial \boldsymbol{M}_{d}}{\partial r_{i}} r_{i r}, \boldsymbol{f}_{r}=\sum_{i=1}^{q} \frac{\partial \boldsymbol{f}_{d}}{\partial r_{i}} r_{i r}, \\
\boldsymbol{x}_{r}=\sum_{i=1}^{q} \frac{\partial \boldsymbol{x}_{d}}{\partial r_{i}} r_{i r}, \dot{\boldsymbol{x}}_{r}=\sum_{i=1}^{q} \frac{\partial \dot{\boldsymbol{x}}_{d}}{\partial r_{i}} r_{i r}, \\
\ddot{\boldsymbol{x}}_{r}=\sum_{i=1}^{q} \frac{\partial \ddot{\boldsymbol{x}}_{d}}{\partial r_{i}} r_{i r}, \boldsymbol{F}_{r}=\sum_{i=1}^{q} \frac{\partial \boldsymbol{F}_{d}}{\partial r_{i}} r_{i r},
\end{gathered}
$$

Substituting Eq. (13) into Eq. (11), yields

$$
\begin{aligned}
& \sum_{i=1}^{q}\left(\boldsymbol{M}_{d} \frac{\partial \ddot{\boldsymbol{x}}_{d}}{\partial r_{i}} r_{i r}+\frac{\partial \boldsymbol{f}_{d}}{\partial r_{i}} r_{i r}\right) \\
= & \sum_{i=1}^{q}\left(\frac{\partial \boldsymbol{F}_{d}}{\partial r_{i}} r_{i r}-\frac{\partial \boldsymbol{M}_{d}}{\partial r_{i}} r_{i r} \ddot{\boldsymbol{x}}_{d}\right)
\end{aligned}
$$

and comparing the coefficient $r_{i r}$, the sensitivity equation is obtained as

$$
\boldsymbol{M}_{d} \frac{\partial \ddot{\boldsymbol{x}}_{d}}{\partial r_{i}}+\frac{\partial \boldsymbol{f}_{d}}{\partial r_{i}}=\frac{\partial \boldsymbol{F}_{d}}{\partial r_{i}}-\frac{\partial \boldsymbol{M}_{d}}{\partial r_{i}} \ddot{\boldsymbol{x}}_{d}
$$

Quantities $\partial \boldsymbol{x}_{d} / \partial r_{i}, \partial \dot{\boldsymbol{x}}_{d} / \partial r_{i}, \partial \ddot{\boldsymbol{x}}_{d} / \partial r_{i}$, can be determined by solving Eq. (15) using numerical methods. Substituting $\partial \boldsymbol{x}_{d} / \partial r_{i}, \partial \dot{\boldsymbol{x}}_{d} / \partial r_{i}, \partial \ddot{\boldsymbol{x}}_{d} / \partial r_{i}$, into Eq. (8), the variances and covariances of the responses can be computed. They are represented as

$$
\begin{aligned}
& \operatorname{Cov}(\boldsymbol{x}) \\
= & \sum_{i=1}^{q} \sum_{j=1}^{q}\left(\frac{\partial \boldsymbol{x}_{d}}{\partial r_{i}}\right)\left(\frac{\partial \boldsymbol{x}_{d}}{\partial r_{j}}\right)^{T} \operatorname{Cov}\left(r_{i}, r_{j}\right), \\
& \operatorname{Cov}(\dot{\boldsymbol{x}}) \\
= & \sum_{i=1}^{q} \sum_{j=1}^{q}\left(\frac{\partial \dot{\boldsymbol{x}}_{d}}{\partial r_{i}}\right)\left(\frac{\partial \dot{\boldsymbol{x}}_{d}}{\partial r_{j}}\right)^{T} \operatorname{Cov}\left(r_{i}, r_{j}\right), \\
& \operatorname{Cov}(\ddot{\boldsymbol{x}}) \\
= & \sum_{i=1}^{q} \sum_{j=1}^{q}\left(\frac{\partial \ddot{\boldsymbol{x}}_{d}}{\partial r_{i}}\right)\left(\frac{\partial \ddot{\boldsymbol{x}}_{d}}{\partial r_{j}}\right)^{T} \operatorname{Cov}\left(r_{i}, r_{j}\right),
\end{aligned}
$$

The mean values, variances and covariances, $E(\boldsymbol{x})$, $E(\dot{\boldsymbol{x}}), E(\ddot{\boldsymbol{x}}), \operatorname{Cov}(\boldsymbol{x}), \operatorname{Cov}(\dot{\boldsymbol{x}}), \operatorname{Cov}(\ddot{\boldsymbol{x}})$, of the random vibration responses, $\boldsymbol{x}, \dot{\boldsymbol{x}}, \ddot{\boldsymbol{x}}$, are obtained from the Eqs (12) and (16). Obviously, the mean values, variances and covariances are accurate to only the first order. Because the results are only good up to the first order, the analysis presented is restrictive, but it can be useful in many practical applications.

\section{Numerical example}

Consider a vibratory machine is modeled as Fig. 1 with $m_{1}=130.66(\mathrm{~kg})$, and $m_{2}=270.72(\mathrm{~kg})$. The random stiffnesses $k_{0}$ and $k$ of springs are mutual independent random variables, its mean value and standard variance are taken as $k_{0}=(50000,2500)(\mathrm{N} / \mathrm{cm})$ and $k=(15680,784)(\mathrm{N} / \mathrm{cm})$. The damping coefficient $c$ is $c=20.5(\mathrm{Ns} / \mathrm{cm})$. The damping and stiffness coefficients $\Delta c$ and $\Delta k$ of interval spring are $\Delta c=10.0(\mathrm{Ns} / \mathrm{cm})$ and $\Delta k=292000(\mathrm{~N} / \mathrm{cm})$. The interval $e$ of interval spring is $e=0.39(\mathrm{~cm})$. The frequency $\omega$ of the main shaft is $\omega=57.5(1 / \mathrm{s})$. The eccentricity $r$ of the main shaft is $r=1.6(\mathrm{~cm})$. The matrix $\boldsymbol{R}$ of the random parameters is $\boldsymbol{R}=\left[k_{0}, k\right]^{T}$, where its joint probability density or distribution functions does not be given, but only the first two moments.

The mean value and standard variance of displacement $x_{1}$ are depicted in Figs 2 and 3, the covariance of displacements $x_{1}$ and $x_{2}$ is depicted in Fig. 4. The Figs 3 and 4 show random variations (standard variance and covariance) with respect to a synthesis influence of 


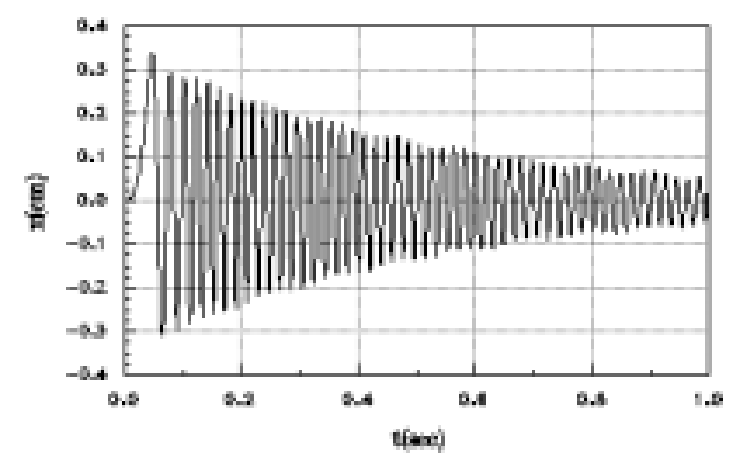

Fig. 2. The mean displacement $x_{1}$.

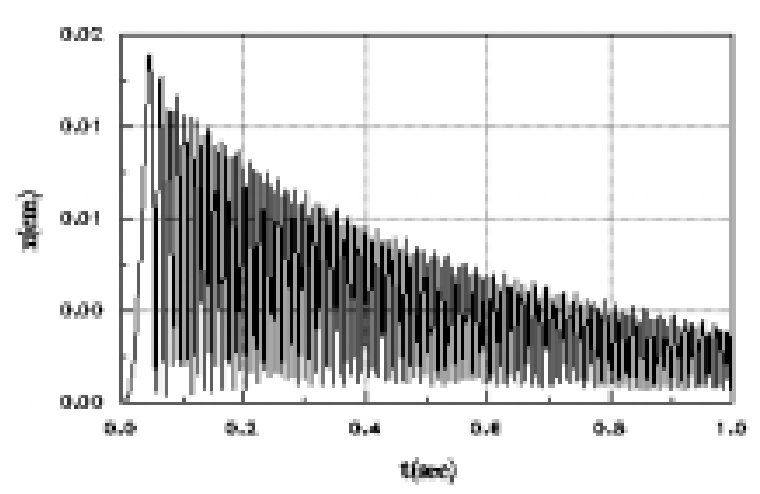

Fig. 3. The standard variance of displacement $x_{1}$.

the random parameters $k_{0}$ and $k$. The each order equations derived earlier are by the implicit Newmark- $\beta$ method.

The calculation of the random responses requires the knowledge of the probability distributions, or the joint distribution. In practice, the available information or data may be sufficient only to evaluate the first and second moments; namely, the mean values, variances and covariances of the respective random variables. Practical measures of responses, therefore, must often be limited to functions of these first two moments. Under this condition, the implementation of responses must necessarily be limited to a formulation based on the first and second moments of the random variables. The method has proven to be efficient in vibratory machines. A major advantage of the technique is that the joint probability density or distribution functions need not be known, but only the first two moments. Besides, Input of independent random parameters can not bring on output of independent random responses in the system with random parameters. The responses of the system are generally correlative, and the theory in the paper educe the same conclusion.

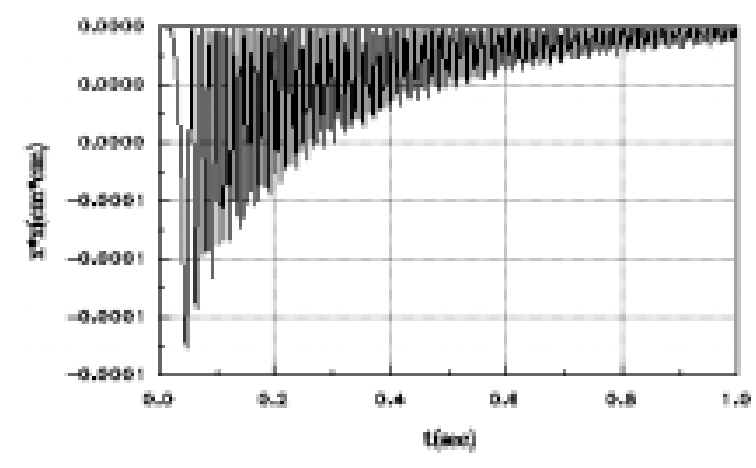

Fig. 4. The covariance of displacements $x_{1}$ and $x_{2}$.

\section{Conclusion}

A vibratory machine with random parameters is usually effected random excitations. Randomness may be mathematically modeled either as a random variable or a stochastic process. Machines with random parameters are complicated to analyze, since the response is statistically correlated to these parameters. This paper bases on the stochastic perturbation technique to systematically investigate the random responses of a vibratory machine. The mechanical development has been obtained. The results obtained are easily amenable to computational procedures.

\section{Acknowledgment}

We would like to express our appreciation to China National Natural Science Foundation (number: 19990510, 50175043), 973 Project Foundation of China (number: 1998020320) and Foundation for University Key Teacher by Ministry of Education for supporting this research.

\section{References}

[1] D.J. Inman, Engineering vibration, Applied Mechanics Reviews 48(6) (1995).

[2] Y.K. Lin, Probabilistic Theory of Structural Dynamics, McGraw-Hill, Inc., New York, 1967.

[3] S.H. Crandall and W.D. Mark, Random Vibration in Mechanical Systems, Accademic Press, Inc., New York, 1963.

[4] A.W. Roberts and W.H. Charlton, Determination of natural responses of mechanical systems using correlation techniques, Experimental Mechanics 15(1) (1975), 17-22.

[5] I.I. Gerega, I.S. Lozovoi, M.R. Kozulkevich and V.M. Shopa, Generalized mathematical-model of the multiple-mass dynamic system of a vibration machine, Soviet Applied Mechanics 27(12) (1991), 1219-1224. 
[6] T.S. Sankar, S.A. Ramu and R. Ganesan, Stochastic finite element analysis for high speed rotors, Journal of Vibration, Acoustics, Stress, and Reliability in Design 115(1) (1993), 5964.

[7] J. Dong, Time series models for vehicle random vibration simulation tests, International Journal of Vehicle Design 16(6) (1995), 581-593.

[8] J. Jerrelind and A. Stensson, Nonlinear dynamics of parts in engineering systems, Chaos, Solitons and Fractals 11(15) (2000), 2413-2428. 



Submit your manuscripts at

http://www.hindawi.com
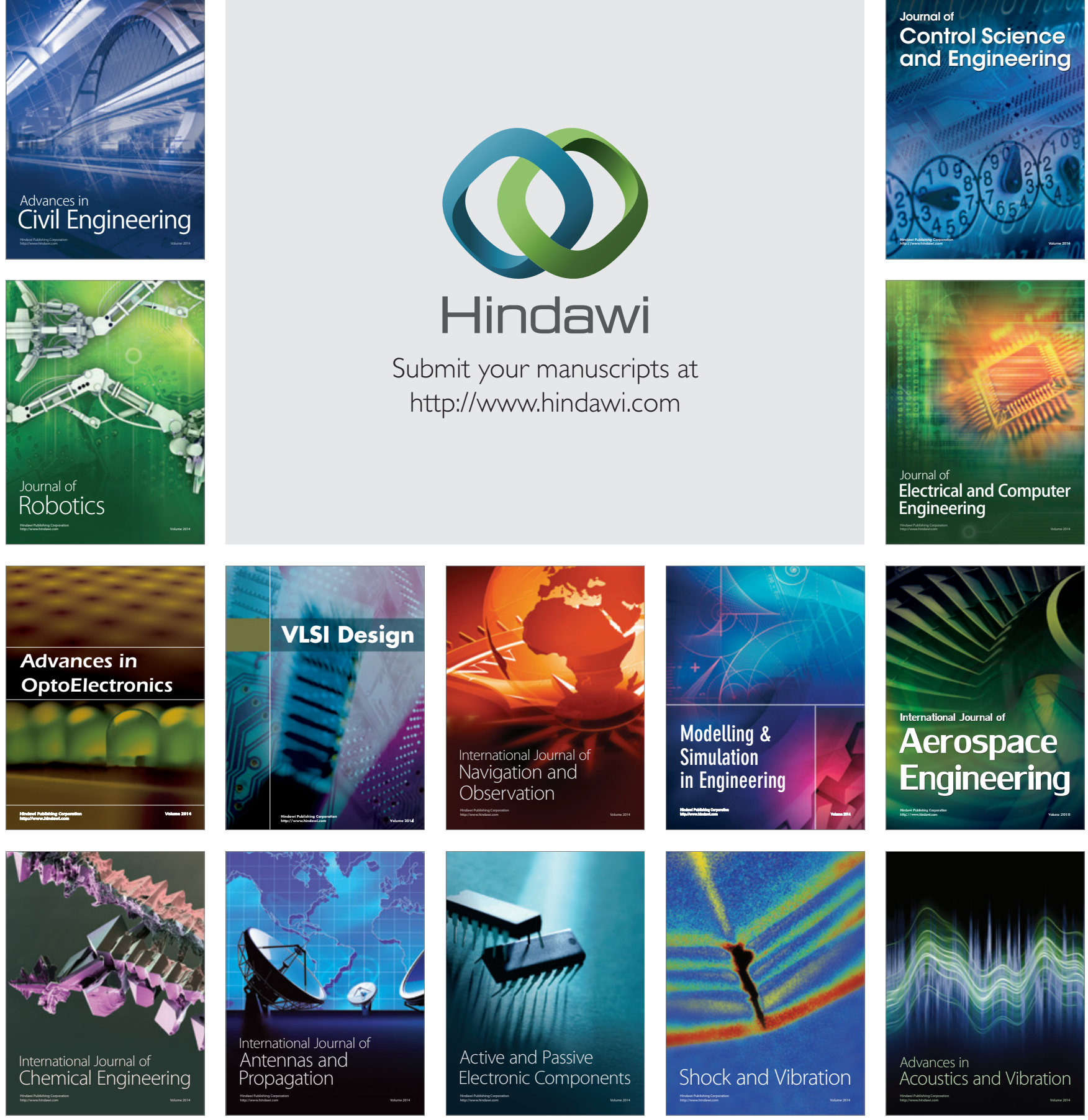